\title{
Fictitious pseudo Meig's syndrome: A medical emergency
}

S. Shukla ${ }^{1}$, N. Acharya ${ }^{2}$, S. Acharya ${ }^{3}$, D.P. Rajput ${ }^{4}$, S. Vagha $^{5}$

${ }^{1}$ Asso. Prof, Dept. of Pathology, ${ }^{2}$ Asso. Prof, Dept. of Obst \& Gynec, ${ }^{3,4}$ Asso. Prof, Dept. of Medicine, ${ }^{5}$ Prof \& Head, Dept. of Pathology, J N Medical College, DMIMS Univ, Sawangi (Meghe), Wardha, M.S

\begin{abstract}
The present case scenario deals with an acute on chronic symptomatology, and collapsed state of the patient with poor vitals on admission in casualty. Clinical work up pointing to an elevated serum CA 125 levels and USG pelvis suggesting peritoneal adhesions with cystic to firm mass in left ovary and minimal free fluid in abdomen and cul de sac, X Ray chest suggesting right sided Pleural effusion.

It was after careful evaluation of the case, with past and present history along with signs, symptommatology and intraoperative findings that differentials like Chronic granulomatous lesions, endometriosis, Neoplastic lesions with metastasis and Meig's syndrome or Pseudo Meig's were evaluated. Finally, a diagnosis of genital tuberculosis with enodmetriosis was confirmed on histopathological evaluation.
\end{abstract}

Key Words: Tubo ovarian mass, fibromyomas, ovarian cysts, endometriosis, Meigs syndrome, Pseudo Meig's.

\section{Introduction}

At times clinical presentation of a medical emergency can be extremely deceptive and difficult to interpret, a particular case may seem to show signs and symptoms which can many a times mislead a clinician. This particular case deals with a $35 \mathrm{yr}$ old female presenting in casualty in a semiconscious state with pain, rebound tenderness, ascites \& pleural effusion. USG of abdomen was done out which revealed a cystic ovarian mass suspicious of malignant nature along with free fluid in peritoneum and cul de sac, and a raised level of CA 125.

Correspondence: S. Shukla

E-mail: samarthshukla@hotmail.com
Present case symptomatology points to extreme spectral ends anything from an ectopic or endometriosis to development of a torsion ovary; even a metastasis cannot be ruled out. The combination of pleural effusion further increases the complexities adding the touch of Meig's syndrome or further specific sub entity of Pseudo Meig syndrome. Considering the past history of the particular case with weight loss, fatigue, malaise, chronic vague abdominal discomfort, and altered bowel habits with low grade fever on and off, chronic inflammatory ailment has to be considered as well. Differentials which should be primarily considered are Endometriosis, ectopic pregnancy, 
genital tuberculosis and or neoplasm not the last, Meig's or pseudo Meig's Syndrome.

Active endometriosis produces inflammatory mediators that cause pain and inflammation, as well as scarring or fibrosis of surrounding tissue. Endometriosis in perimenopausal age group is not uncommon an entity and is characterized by pain in the lower abdominal or pelvic area during periods. Ectopic rest of endometrium in endometriosis can be commonly seen anywhere from the periuterine adenexa to internal viscera as well as in form of peritoneal implants and rarely in the diaphragm and lungs. Symptoms and signs basically will depend on the site of endometriotic rest. Endometriosis is never curable though symptoms can be managed to some extent. It usually wanes off after menopause. Endometriosis can present from a nonspecific vague pain in the lower pelvic quadrants to extreme tenderness and pain radiating to the lower abdomen and back and very disturbing for patients. Additionally other lesions may be present notably, endometriomas of the ovary, scar formation, and peritoneal defects or pockets. Endometrioma on the ovary of any significant size (Approx. $2 \mathrm{~cm}+$ ) must be removed surgically because hormonal treatment alone will not remove the full endometrioma cyst, which can progress to acute pain from the rupturing of the cyst and internal bleeding. Endometrioma is sometimes misdiagnosed as ovarian cysts.

Genital tuberculosis is by no means a rarity at least not in the females of the developing world though it has dwindled to a proportionate extent in the Western hemisphere (developed states). There is little correlation between presenting complaints and physical findings in genital TB. About $35-50 \%$ of patients have an entirely normal examination., 2 Literature on genital TB appear to agree that the fallopian tubes are likely the initial source of infection because both tubes are involved in nearly $100 \%$ of cases, ${ }^{3-5}$ form the primary targets from where the peritonitis and severe or massive adhesions can originate. Tuberculous peritonitis is seen in combination with female genital tract $\mathrm{TB}$ in approximately $45 \%$ cases and is thought to be responsible for the often extensive adhesions seen in patients with pelvic TB. ${ }^{6}$ Two types of tuberculous peritonitis have been described: the plastic variety and the serous variety.. With peritonitis, an associated pleural effusion is not uncommon, although most patients have no parenchymal abnormalities on chest radiograph. The intense adhesions in the peritoneum involves the omentum and the intestinal viscera along with the uterine adenexa resulting in situations mimicking malignant ascites or pseudomyxoma peritonii.

Meigs syndrome is defined as the triad of benign ovarian tumor with ascites and pleural effusion that resolves after resection of the tumor. The ovarian tumor in Meigs syndrome is a fibroma. PseudoMeigs syndrome consists of pleural effusion, ascites, and benign tumors of the ovary other than fibromas. These benign tumors include those of the fallopian tube or uterus and mature teratomas, struma ovarii, and ovarian leiomyomas ${ }^{7}$ This terminology sometimes also includes ovarian or metastatic gastrointestinal malignancies. Atypical Meigs characterized by a benign pelvic mass with right-sided pleural effusion but without ascites has 
been reported at least twice. The present case has to be cautious evaluated for ruling out Meig's as well as Pseudo meig's syndrome.

Taking into account the ambiguous clinical case scenario with an emergency situation in managing the patient, the case becomes particularly interesting in the sense that it presents with an acute as well as chronic signs and symptoms, and is interpretatively it extremely hazy; the findings do not contribute completely to differentiate whether it is malignant benign or chronic inflammatory condition. It is only by meticulous histopathological evalution and interpretation that the whole puzzled case scenario falls into place.

\section{Case report}

35 yr old female $\mathrm{G}_{2} \mathrm{P}_{2}$ was received at the casualty in a mentally obtunded state. She was not oriented to time, place or person and her vitals were extremely altered. She was immediately assessed for her cardiopulmonary status, her breathing and ventilation status were verified she was found to have a clear airway with a normal pattern of respiration (audible as well as visible), though rapid. Due to depressed consciousness she had a risk of hypoventilation as well as aspiration. Her ventilation was closely monitored. She had a BP of $80 / 40 \mathrm{mmHg}$, with a low volume rapid pulse of 160 beats/min. her respiration was 34/min. Urgent ECG was done which showed sinus tachycardia. Ryle's tube was inserted 20 cc of non hemorrhagic non bile stained fluid aspirated and sent for cytopathological evaluation. Once it was as certained that the cardiopulmonary status was clear hypoglycemia and hypoventilation the much anticipated but treatable clinical complications. As a general rule, despite availability of pulsemeter a general delay in the $\mathrm{pO}_{2}$ and $\mathrm{pH}$ reports is anticipated hence, $100 \%$ oxygen by face mask was administered to the patient. Parenteral lines were established, emergency lab investigationsHaemogram, clinical chemistry for blood gases, plasma glucose (random), serum electrolytes $\left(\mathrm{Na}^{+}, \mathrm{K}^{+}, \mathrm{Cl}^{-}, \mathrm{HCO}_{3}^{-}\right)$Serum urea, creatinine, BUN , AST, ALT, Alkaline Phosphatase, CA 125 levels were carried out. Immediately thereafter $200 \mathrm{ml}$ of normal saline was given rapidly but BP did not come up, so IV dopamine drip was started at a rate of 10 micrograms $/ \mathrm{kg} /$ minute. $50 \mathrm{ml}$ iv bolus of $50 \%$ dextrose solution was started after sending. She was catherized; appearance of urine was unremarkable (negative for gross haematuria). Her past history from relatives revealed Loss of appetite, malaise, weakness and weight loss. she had severe abdominal pain and distention since last 5 days, she had altered bowel and bladder habit with vague pain during defecation since 3 months, she also had chronic dysmenorrhea though the duration of the same was uncertain.

$\mathrm{O} / \mathrm{E}$ she had pallor, but there was no evidence of no icterus, cyanosis, clubbing or edema, she had a temp of $38^{\circ} \mathrm{C}$ Systemic examination: Abdominal inspection revealed fullness of lower abdomen without any prominent veins, visible peristalsis or distortion of the umbilicus. Per abdominal examination showed rebound tenderness, along with guarding and rigidity. There were signs of free fluid. Liver, spleen, and inguinal lymph nodes were not palpable, hernial orifices were normal. On percussion there was shifting dullness due to free fluid. USG abdomen (bed side ) was called for .CVS examination unremarkable - ECG showed 
sinus tachycardia. RS examination revealed signs of right sided pleural effusion, $\mathrm{X}$ ray chest $\mathrm{PA}$ view was done. Considering her altered consciousness CNS examination was carried out, Glasgow coma scale turned out to be 8 , brain stem signs were normal, she was moving all her limbs to painful stimuli, deep tendon reflexes were normal, bilateral planters were flexors, neck was supple to passive flexion.

Her lab results were as follows: Haemogram revealed a $\mathrm{Hb}$ of $10 \mathrm{gm} \%$ and $\mathrm{PCV}$ of $33 \%$, pointing towards an acute blood loss, WBCLeukocytosis( lymphoctyosis) TLC $-15,000 / \mathrm{mm}^{3}$ DLC- 46\% Polymorphs, 2\%Eosinophils, 2\%Monocytes, 50\% Lymphocytes. Urine microscopy was negative for haematuria and casts as well as crystals, Urine pregnancy test was negative. Her clinical chemistry- electrolytes, enzymes were within normal limits (AST being mildly over the limits 65 IU though insignificant). CA 125 levels were raised to $150 \mathrm{IU}$, a hint towards peritonism or uterine adenexal pathology. Her blood gas analysis was suggestive of respiratory alkalosis. Ultrasound abdomen revealed free fluid in peritoneum (approximately 500ml) and cul de sac along with cystic to partially solid mass in the left ovary. CT scan of brain was normal, other visceral structures were unremarkable. X Ray chest PA view was suggestive of minimal fluid in the right pleural cavity, There was no gas under the diaphragm (for perforation peritonitis)

Her presentation as a surgical emergency ( acute abdomen and left sided adenexal mass and an elevated CA $125 \mathrm{IU}$ ) prompted an exploratory laparotomy under general anesthesia. On opening the abdomen by right infraumbilical paramedian incision, plenty of deep straw colored fluid came out of the peritoneal cavity. It was sent for cytological examination. A 6 x $4 \mathrm{~cm}$ sized cystic hemorrhagic ovarian mass arising from the left ovary with twisted pedicle and adherent omentum was detected. Uterus was normal in size and the other ovary was slightly larger than normal. A feature of unexpected but extreme significance was the present of tubercles all over the uterine and bilateral adenexa (Fig-1)Yet, the haemorraghic, partial solid to cystic nature of ovarian mass with adhesions could not be undermined for neoplastic behavior. Considering the acute nature of onset , suspicious cystic mass in leaft ovary along with the parity and age a decision of total abdominal hysterectomy and bilateral salpingo oophorectomy was taken, for which prior consent was taken. Exploration of the peritoneal cavity did not show any palpable lymph node or other metastasis. One unit of blood was transfused during surgery.

Surgical Pathological and cytopathological analysis were as follows- peritoneal fluid was suggestive of Gross: Cob web formation, exudative character, with predominant lymphocytosis Surgical pathology and sampling was done histopathological report was suggestive of genital tuberculosis (tubercular endometritis (Fig-2), tubercular salphigo opheritis (Fig-3). and perioopheritis) with endometriosis in the 1t. fallopian tube (Fig-4)., perifimbrial tissue (Fig-5). and ruptured chocolate cyst in the left. ovary. There was no evidence of malignancy on histopathology. 


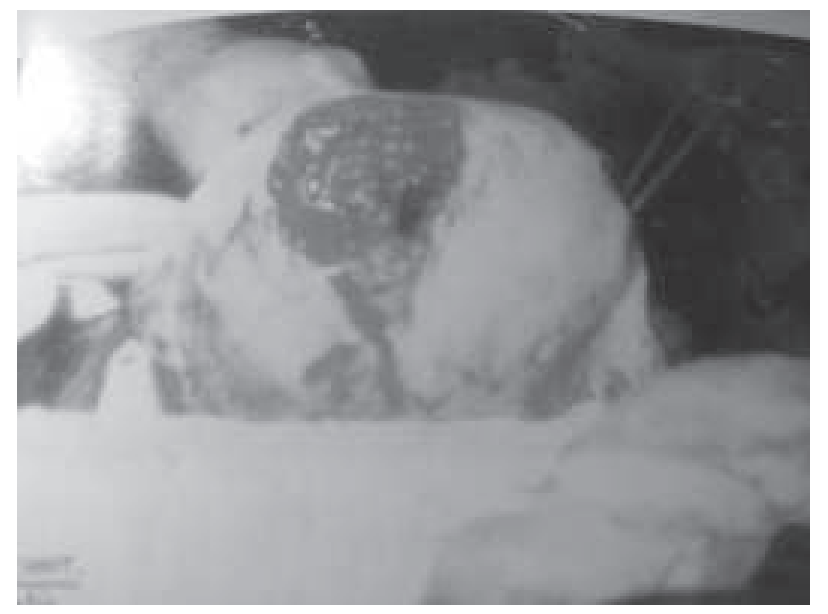

Fig 1: depicting Gross Uterus with bilateral adenexa (It. ovary showing hemorrhagic cystic mass, while the uterine serosa and the rt. Ovary showing tubercles)

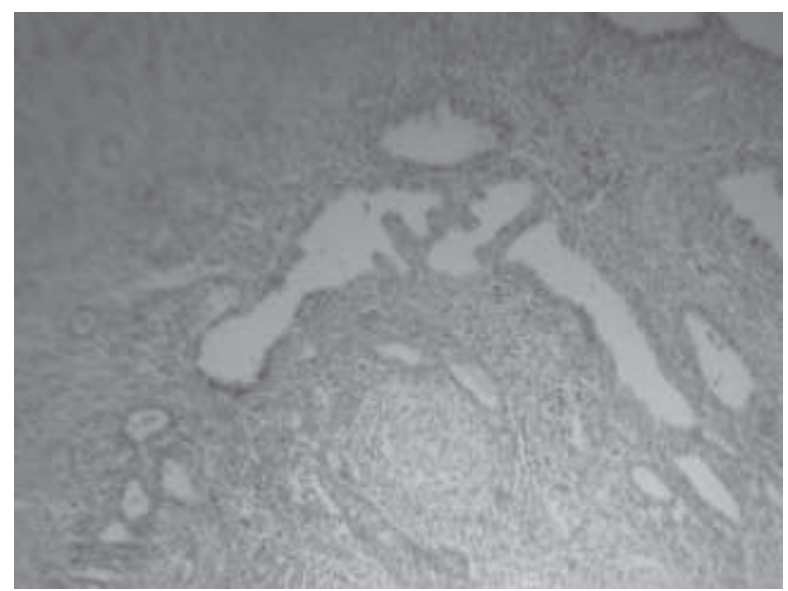

Fig 2: H\&E stained slide from endometrium showing endometrial tissue with chronic tubercular granulomatous changes

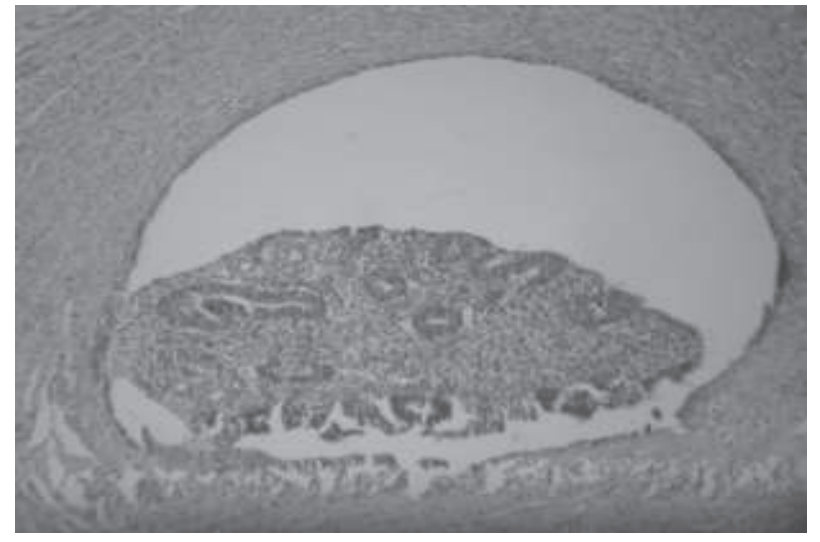

Fig 3: H\&E stained slide showing Extensive granulomatous changes (tubercular) with giant cell formation in the Tubo ovarian stroma.

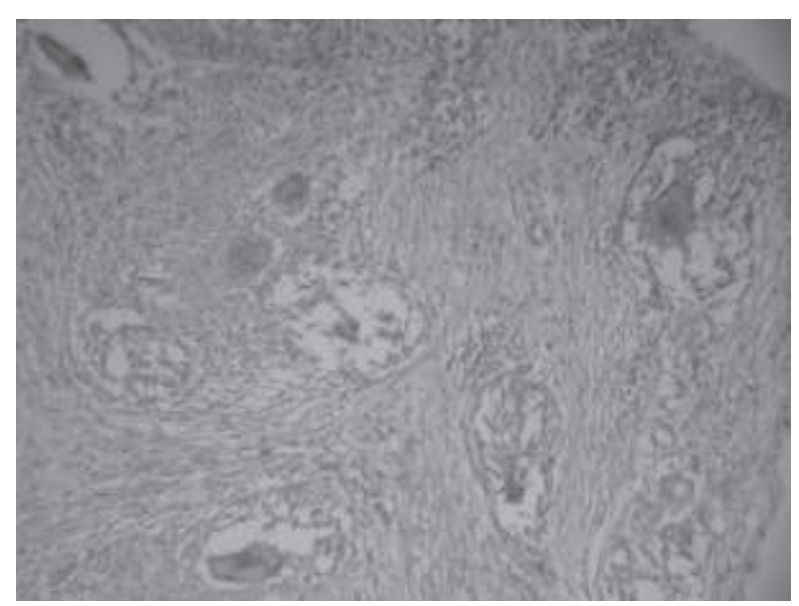

Fig 4: H\&E stained slide showing extensive endometriosis in the tubal lumen (ectopic rest of endometrial glands and stroma in the Fallopian tube) 


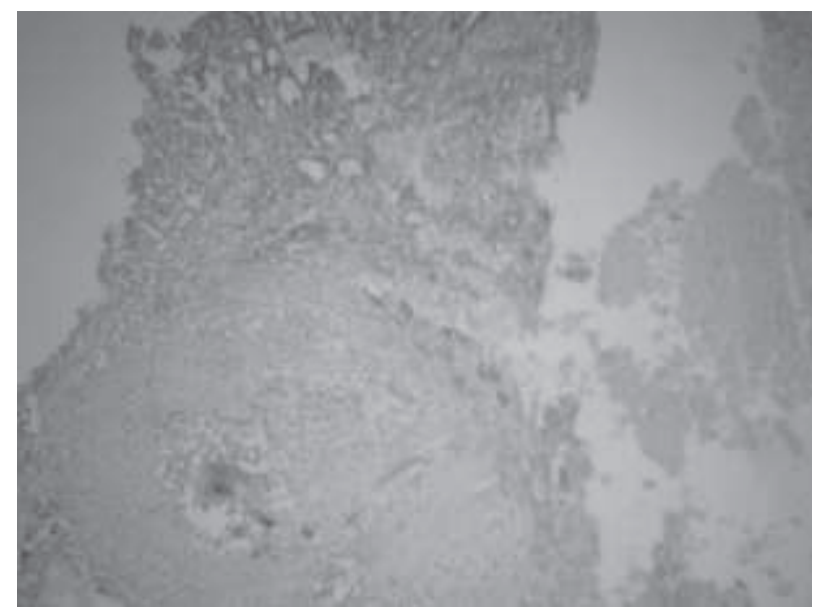

Fig 5 H\&E stained slide showing Perifrimbrial stromal tissue with tubercular grnaulomas as well as extensive endometrial rests.

\section{Discussion}

In the given case the initial presentation in the casualty was shock with complaints of acute abdominal pain. Acute abdomen presenting in a young female as medical emergency, with clinical signs of shock can turn out to entertain a host of differentials. An array of differential diagnosis have to be considered as part and parcel in a young female which includes gynaec and non gynaec (surgical) causes of acute lower abdomen pain . Most important gynaec causes include Ectopic pregnancy, Torsion of ovary, abortion, endometriosis, dysfunctional uterine bleeding, pelvic inflammatory disease and fibroids. As far as the surgical cause is concerned the differentials include appendicitis, perforation peritonitis, renal colic, intestinal obstruction.

The patient presenting with acute abdominal tenderness was primarily evaluated for appendicitis, peritonitis and intestinal obstruction clinically and the signs of guarding rigidity with rebound tenderness were indicative of all three. With no history of vomiting or visible peristalsis on abdominal examination, intestinal obstruction became a less likely possibility. There was no history of renal colic (as given by pt's relative) and findings of guarding rigidity with rebound tenderness virtually set aside colicky nature of visceral pain (mainly pertaining to renal colic). $\mathrm{X}$ Ray abdomen as well as chest X-ray PA view were done. Gas under diaphragm was absent though as expected there was presence of minimal Rt sided pleural effusion . The urinary examination/USG was negative for haematuria as well as crystals/casts ruling out Urinary tract pathology. The negative UPT test was extremely helpful in eliminating ectopic pregnancy. Pelvic examination was rather unremakable for uterus, but that did not aid in eliminating gyanecological pathology of endometriosis though differentials of fibroids, pelvic inflammatory disease were less likely. On $\mathrm{PV}$ examination uterus was difficult to manipulate or move and was suggestive of adhesions, even the cul de sac was not evidently palpated (fluid +ve on USG) The ovaries were bilaterally were enlarged on pelvic examination, though final comment was reserved due to presence of ascites (as confirmed on USG).

A differential considered of grave importance was Meigs and Pseudo Meig's syndrome (pleural effusion, ascitis and ovarian mass). The syndrome showing not only very remarkable clinical signs but also giving an extremely significant past history of loss of appetite, malaise, weakness and weight loss promted the clinicians to think in lines of Meig/ 
Pseudo meig syndrome though chronic inflammatory disease was also not ruled out. Considering the clinical emergency and poor vitals of the patient a diagnostic laparotomy was performed and the intraoperative findings correlated with the clinical as well as the USG reports but to add to the dilemma there was extensive spread of tubercles all over the uterine serosa as well as the bilateral adenexa, thereby prompting to entertain a diagnosis of secondary tuberculosis.

The clinical diagnosis of genital TB requires a high index of suspicion. About $20 \%$ of patients with genital TB give a history of TB in their immediate family. ${ }^{3}$ Tuberculosis rarely spares any particular organ and is extremely contagious as far as the internal milieu of the human viscera is concerned. The mode of spread from primary site is usually hematogenous (lung) or lymphatic (intestinal) and occasionally occurs by way of direct contiguity with an intraabdominal or peritoneal focus. ${ }^{3,8}$

Endometriosis can affect any woman, from premenarche to postmenopause, regardless of her race or ethnicity or whether or not she has had children. It is primarily a disease of the reproductive years. Estimates about its prevalence vary, but 5$10 \%$ is a reasonable number, more common in women with infertility (20-50\%) and women with chronic pelvic pain ( $80 \%$ ). ${ }^{9}$ As it is an estrogendependent process, it can persist beyond menopause and persists in up to $40 \%$ of patients following hysterectomy. ${ }^{10}$ A major symptom of endometriosis is recurring pelvic pain.. Some women will have little or no pain despite having extensive endometriosis affecting large areas or having endometriosis with scarring. On the other hand, they may have severe pain despite few small areas of endometriosis. However, pain does typically worsen with severity.

The intra operative findings in this particular case were ascites peritoneal hemorrhage with loose adhesions in the peritoneum, tubercles in the uterine serosa as well as bilateral adenexa and omentum. The gross findings were characteristic of a chronic granulomatous lesion more specifically, tuberculosis. Besides this another important gross finding which was present was the left. sided cystic to haemorrahgic firm ovarian mass highly suspicious of neoplastic character. However the histopathology of hysterectomy with bilateral salphingo opherectomy specimen was suggestive of genital tuberculosis and goes well with the patients chronic complaints as well as ascitis and pleural effusion (ruling out Meig's as well as Pseudo Meig's Syndrome). The cystic to firm mass of right ovary was diagnostic of chocolate cyst (endometrioma) with presence of florid endometriosis which was present in this particular case, mostely in the perifimbrial adenexa as well as in fallopian tube lumen and ovary.

While women with ovarian cancer often have an elevated level of CA 125, an elevated CA 125 level doesn't always mean you have ovarian cancer. Some women with ovarian cancer never have an elevated CA 125 level. Many other conditions also can cause an elevated CA 125 level, including: diverticulitis, endometriosis, liver cirrhosis, normal menstruation, pregnancy, uterine fibroids, peritoneal Inflammation/Irritation(peritonism). For these reasons CA 125 testing in women with an average risk of ovarian cancer should not be set as an mandatory diagnostic indication. 


\section{Conclusion}

Case scenario jigsaw now seems to fall into place. With findings of genital tuberculosis and endometriosis overlapping in the clinical symptomatology, along with the chronic effusions and peritoneal adhesions, altered bowel habits, chronic dysmennorrhoea and vague pain; acute exacerbation of pain in the left adenexa due to ruptured chocolate cyst resulting in acute peritonism (clinically as shock) symptoms mimicked Meig's syndrome or Pseudo Meig's. The raised CA 125 levels can now be attributed to peritoneal reaction rather than an ovarian mass. With aids of histopathological diagnosis the clinically deceptive picture became very clear of a case of that of genital tuberculosis concomitant with endometriosis. Many clinicians have come across such clinical presentation but the sole intention of the case report is to exemplify the case scenario to reiterate the fact that multiple pathological lesions are not uncommon in female genital system and should be duely considered before forming any sort of rigid or dogmatic opinions on features like effusions, adenexal masses and a raised CA 125.

\section{References}

1. H.B. Simon, A.J. Weinstein, M.S. Pasternak, et al: Genitourinary tuberculosis: Clinical features in a general hospital population. Am J Med 1977;63:410.
2. A.M. Sutherland: Postmenopausal tuberculosis of the female genital tract. Obstet Gynecol 1982;59:549.

3. G. Schaefer: Female genital tuberculosis. Clin Obstet Gynecol 1976;19:223.

4. F. Nogales-Ortiz, I. Tarancion, F.F. Jr. Nogales: The pathology of female genital tuberculosis: A 31-year study of 1436 cases. Obstet Gynecol 1979;53:422.

5. O.F. Saracoglu, et al: Pelvic Tuberculosis. Int J Gynaecol Obstet 1992;37:115.

6. G. Schaefer: Tuberculosis of the female genital tract. Clin Obstet Gynecol 1970;13:965

7. J.S. Jr. Dunn, C.D. Anderson, M.W. Method. Hydropic degenerating leiomyoma presenting as pseudo-Meigs syndrome with elevated CA 125. Obstet Gynecol. Oct 1998;92(4):648-9.

8. A.G. Goldin, W.T. Baker: Tuberculosis of the female genital tract. J Ky Med Assoc 1985;83:75.

9. "Endometriosis cancer risk". medicalnewstoday.com. 5 July 2003. http://www.medicalnewstoday.com/ medicalnews.php?newsid=3890. Retrieved 2007;07- 03 .

10. Roberts, Michelle (3 July 2007). "Endometriosis 'ups cancer risk'". BBC News. BBC /news.bbc.co.uk. http:// news.bbc.co.uk/2/hi/health/6262140.stm. Retrieved 2007;07-03. 